\title{
Adhesion of Irradiated Diazoquinone-Novolac Photoresist Films to Single-Crystal Silicon
}

\author{
S. A. Vabishchevich ${ }^{a}$, S. D. Brinkevich ${ }^{b}$ * , N. V. Vabishchevich ${ }^{a}$, D. I. Brinkevich ${ }^{b}$, and V. S. Prosolovich ${ }^{b}$ \\ ${ }^{a}$ Polotsk State University, Novopolotsk, 214400 Belarus \\ ${ }^{b}$ Belarussian State University, Minsk, 220050 Belarus \\ *e-mail: brinkevichsd@bsu.by
}

Received June 2, 2021; revised July 2, 2021; accepted July 6, 2021

\begin{abstract}
In this work, the effect of $\gamma$-irradiation on the adhesion properties of FP9120 diazoquinonenovolac photoresist films deposited on single-crystal silicon wafers by centrifugation was studied using an indentation method. It was found that $\gamma$-irradiation led to a decrease in the specific peeling energy $G$ of photoresist films on silicon. In this case, the IR spectra of the photoresist exhibited a decrease in the intensity of vibration bands due to the $\mathrm{Si}-\mathrm{O}-\mathrm{C}$ moiety, which is responsible for adhesion to silicon, in the course of $\gamma$ irradiation. The observed experimental results were explained taking into account the radiation-chemical and relaxation processes occurring both at the photoresist/silicon interface and in the bulk of the polymer film.
\end{abstract}

Keywords: diazoquinone-novolac photoresist, $\gamma$-irradiation, adhesion, silicon

DOI: $10.1134 / \mathrm{S} 0018143921060151$

\section{INTRODUCTION}

Modern processes for the fabrication of electronic products are based on the use of photolithography (PL) - a method of forming a given pattern on a silicon substrate to obtain the required microcircuit topology [1]. The PL operations are repeated many times in the course of manufacturing a microcircuit on a plate. Thus, up to 10 photolithography operations are used in the formation of hardware components based on $n$ MOS technology, whereas about 22 PL operations are used in BiCMOS technology [2].

Resists-chemical substances or their mixtures that change their physicochemical properties under the influence of high-energy radiation (ultraviolet light, $\mathrm{X}$-rays, and electron or ion beams) - are the materials used in lithographic processes. Positive two-component photoresists (PRs) based on photosensitive $o$-naphthoquinone diazide and a novolac resin base are most widely used in technological microelectron- ics processes. Under the influence of radiation with a wavelength $\lambda$ of $\sim 300-350 \mathrm{~nm}, o$-naphthoquinone diazide in the photoresist film undergoes denitrogenation and then converts into $1-H$-indene-3-carboxylic acid (reaction (1)) due to the presence of $1-2 \%$ water in the film. As a result of this photochemical process, the irradiated regions of the photoresist become soluble in a $0.1-0.3 \mathrm{M}$ alkaline developer $[1,2]$.

The FP9120 positive photoresist, which is a composite of $o$-naphthoquinone diazide and a mixture of phenol- and cresol-formaldehyde resins, is widely used in modern semiconductor electronics as a protective photosensitive material in precision photolithographic processes for the manufacture of semiconductor devices and integrated circuits [2]. One of the most important technological characteristics of photoresist films is their adhesion to a single-crystal silicon substrate.<smiles>[R]c1cccc2c1CC=C2C(=O)O</smiles> 